\title{
Calculation of Added Mass for Underwater Vehicles Based on FVM
}

\author{
Lihua SONG \\ No. 710 R\&D Institute, CSIC, Yichang 443003, China \\ Email:song_lh26@126.com
}

Keywords: added mass; finite volume method; unstructured

\begin{abstract}
Based on the solution of Laplace equation in parallel using FVM with unstructured hybrid mesh, the paper studies the calculation of added mass for underwater vehicle. The method is accurate and efficient, which is applicable to both simple shape bodies and complex shape bodies with large curvature and sharp edges. First, the added mass of sphere is calculated using two grids, tetrahedron grid and tetrahedron/prism hybrid grid. It is shown that the calculation results agree with the theoretical results very well. Then, as an engineering application, the added mass coefficients of a suspended platform in unbounded domain are calculated, it is verified in the underwater sea trial.
\end{abstract}

\section{Introduction}

The fluid force of vehicles is decomposed into position force, damping force and inertial force in sailing mechanics, in which position force and damping force can be get by solution of the viscous fluid dynamics equations at different attack angles, sideslip angles and dimensionless rotational angular velocities. The calculation of inertial force, also known as the added mass force, will directly affect the simulation of fluid dynamics for vehicles, thus affecting the calculation of the ballistic equations, and ultimately affect the navigation guidance and control of vehicles. However, the existing calculation methods of added mass is not efficient and the application is limited. It is necessary to study an accurate and efficient calculation method of added mass for complex shape bodies.

Currently, the added mass is calculated by the Hess-Smith method [1,2], which is based on the solution of Laplace equation using the finite element method. Hess-Smith method divides the body surface into $\mathrm{N}$ equal quadrilateral plane elements, assuming that the source intension of each element is uniformly distributed. Hess-Smith method is widely used to calculate the added mass of vehicles in unbounded fluid. However, the Hess-Smith method using traditional boundary element formula needs to solve as much as thousands of elements for small-scale models[3], and this means it need much time to calculate the added mass by the Hess-Smith method. In addition, the Hess-Smith method would be more difficult to deal with the complex shape bodies when the bodies have a large curvature and sharp corners $[4,5,6]$. In fact, the calculation of added mass using the boundary element method is in continuous improvement with the development of the theory of boundary element. For example, zhiliang Lin [7] used FMBEM (fast multiple boundary element method) to calculate the added mass coefficient of suboff model in unbounded fluid.

Based on the method of discrete domain, FEM (finite element method) can be used to calculate the added mass of complex shape bodies in any complex boundary conditions, such as a bounded fluid with wall or free surface boundary. For a bounded fluid, the results of unbounded fluid can be also effective when the object is far enough from the boundary, but when the distance is closer, the added mass will be a significant change, and the near-wall effects must be considered. Many scholars use finite element method to deal with the near-wall effects, solving basic Laplace velocity potential equation to get the added mass of complex three dimensional shape bodies. Li [8] studied the added mass for ship entering and leaving a ship-box with twenty joint elements.

Different with FEM approximation point by point, the FVM (finite volume method) is integral average approximation on the control body to ensure that the physical conservation laws, and can also be applied to any complex boundary and boundary conditions with less calculation than FEM, so FVM is currently the main discrete method in Computational Fluid Dynamics (CFD). From the 
viewpoint of computational fluid dynamics, basic Laplace velocity potential equation is actually the scalar transport equation without convective items, time and source terms, leaving only the diffusion term. In CFD finite volume method, the diffusion terms of the scalar transport equation are all using the second order discrete form of the center, which is very mature discrete. The regional block parallel algorithm based on MPI is the main means of improving computational efficiency in CFD. Therefore, the paper proposes a method to calculate the added mass for underwater vehicle based on the solution of Laplace equation in parallel using FVM. By studying the added mass coefficients of a sphere and a platform suspending in infinite domain, it shows that this method can not only calculate the complex shape of the object additional mass in complex boundary conditions, but also has high computational efficiency.

\section{Velocity potential equation}

Assume $\varphi$ is a scalar function, according to field theory, $\nabla \times \nabla \varphi=0$.If the fluid is irrotational, then $\nabla \times V=0$. So for irrotational flow, there must be a scalar function $\varphi, \nabla \varphi=V$. Scalar function $\varphi$ is potential function of velocity $\mathrm{V}$. If the fluid is incompressible, then $\nabla \cdot \mathrm{V}=0$. So, for incompressible irrotational flow, the potential function of velocity $\mathrm{V}, \varphi$ meets

$$
\nabla \cdot \mathbf{V}=\nabla \cdot \nabla \varphi=\nabla^{2} \varphi=0
$$

According to the physical meaning, $\varphi$ at infinity and object plane should

$$
\lim _{r \rightarrow \infty} \nabla \varphi=0, \frac{\partial \varphi}{\partial n}=V_{n}
$$

$V_{n}$ is the projection of the object surface particle velocity on the local normal direction $n$.

$$
\begin{aligned}
\frac{\partial \varphi}{\partial n} & =\mathbf{n} \cdot \nabla \varphi=\mathbf{n} \cdot \mathbf{V}=V_{n}=\mathbf{n} \cdot \mathbf{V}_{0}+\mathbf{n} \cdot(\boldsymbol{\omega} \times \mathbf{r})=\mathbf{n} \cdot \mathbf{V}_{0}+(\mathbf{r} \times \mathbf{n}) \cdot \boldsymbol{\omega} \\
& =\mathrm{u}_{0} n_{x}+v_{0} n_{y}+w_{0} n_{z}+\omega_{x}\left(y n_{z}-z n_{y}\right)+\omega_{y}\left(z n_{x}-x n_{z}\right)+\omega_{z}\left(x n_{y}-y n_{x}\right)
\end{aligned}
$$

As the Laplace operator is a linear operator, the velocity potential $\varphi$ can be a linear combination of six basic velocity potential $\varphi_{i}$.

$$
\begin{aligned}
& \varphi=u_{0} \varphi_{1}+v_{0} \varphi_{2}+w_{0} \varphi_{3}+\omega_{x} \varphi_{4}+\omega_{y} \varphi_{5}+\omega_{z} \varphi_{6} \\
& \nabla^{2} \varphi_{i}=0, \frac{\partial \varphi_{i}}{\partial n}=N_{i}, \lim _{r \rightarrow \infty} \nabla \varphi_{i}=0 \quad i=1,2,3,4,5,6
\end{aligned}
$$

$\mathbf{N}=\left(\begin{array}{llllll}n_{x} & n_{y} & n_{z} & y n_{z}-z n_{y} \quad z n_{x}-x n_{z} \quad x n_{y}-y n_{x}\end{array}\right) . \varphi_{1} 、 \varphi_{2} 、 \varphi_{3}$ is velocity potential in the $x$-direction $、 y$-direction and $z$-direction respectively in unit speed, $\varphi_{4} 、 \varphi_{5} 、 \varphi_{6}$ is velocity potential around $x$-axis $y$-axis $z$-axis respectively in unit rotation speed.

When the boundary is solid wall or free surface, the boundary condition is as follows:

$$
\frac{\partial \varphi_{i}}{\partial n}=0
$$

The above formula deals with the free surface as the solid wall boundary, and this simplification is rationality and practicability [9]. If you really need to consider free surface condition accurately, the object movement must be linear through modal decomposition. Free surface control equation is linear based on Small amplitude hypothesis, linear boundary condition of free surface and sommerfield radiation boundary condition of far-field is as follows: 


$$
\frac{\partial \varphi_{i}}{\partial z}-\left.\frac{\omega^{2}}{g} \varphi_{i}\right|_{z=0}=0, \lim _{r \rightarrow \infty} \sqrt{r}\left(\frac{\partial \varphi_{i}}{\partial r}-i k \varphi_{i}\right)=0
$$

\section{Numerical methods}

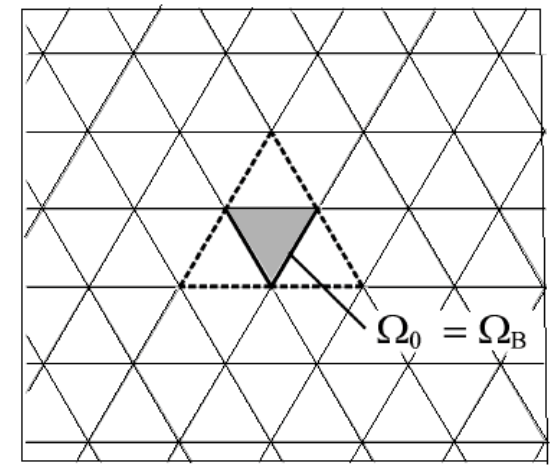

Fig.1. Heart-shaped finite volume template

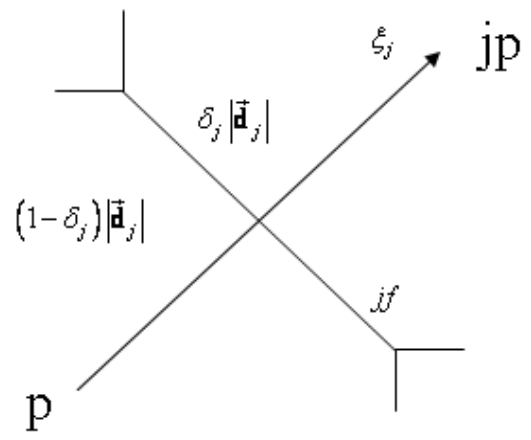

Fig.2. Local coordinate system of interface -jf on control body $\mathrm{P}$

Grid centered finite volume control is adapted as above figure. With gauss formula, we can get the integral equation of Laplace Eq.5 on control volume $\Omega$.

$$
\begin{aligned}
& \oint_{S} \nabla \phi \cdot \mathbf{n} d s=0 \\
& \sum(\nabla \varphi)_{f} \cdot \mathbf{S}_{f}=0 \\
& (\nabla \varphi)_{f} \cdot \mathbf{S}_{f}=\left(\varphi_{j P}-\varphi_{P}\right) \frac{\mathbf{S}_{f} \cdot \mathbf{S}_{f}}{\mathbf{S}_{f} \cdot \mathbf{d}_{f}}+(\overline{\nabla \varphi})_{f} \cdot\left(\kappa_{f}^{x} \mathbf{i}+\kappa_{f}^{y} \mathbf{j}+\kappa_{f}^{z} \mathbf{k}\right) \\
& (\overline{\nabla \varphi})_{f}=\delta{ }_{f}(\nabla)_{P}+\left(1-\delta_{f}\right)(\nabla \varphi)_{j P} \\
& \left.\boldsymbol{\kappa}_{f}^{\prime} \varphi_{P}=\sum \hat{\mathbf{n}}_{f}-(\gamma \hat{\mathbf{d}})_{f}\right) S_{f}=\kappa_{f}^{x} \mathbf{i}+\kappa_{f}^{y} \mathbf{j}+\kappa_{f}^{z} \mathbf{k}, \gamma_{f}=\frac{1}{\hat{\mathbf{n}}_{f} \cdot \hat{\mathbf{d}}_{f}}=\frac{S_{f} d_{f}}{\mathbf{S}_{f} \cdot \mathbf{d}_{f}} \\
& a_{P}^{\prime}=\sum \frac{\mathbf{S}_{f} \cdot \mathbf{S}_{f}}{\mathbf{S}_{f} \cdot \mathbf{d}_{f}}, b_{j P}=\frac{\mathbf{S}_{f} \cdot \mathbf{S}_{f}}{\mathbf{S}_{f} \cdot \mathbf{d}_{f}}, c^{\prime}=\sum(\overline{\nabla \varphi})_{f} \cdot\left(\kappa_{f}^{x} \mathbf{i}+\kappa_{f}^{y} \mathbf{j}+\kappa_{f}^{z} \mathbf{k}\right) \\
& \frac{a_{P}^{\prime}}{\alpha} \varphi_{P}=\sum b_{j P} \varphi_{j P}+c^{\prime}+\frac{a_{P}^{\prime}}{\alpha}(1-\alpha) \varphi_{P}^{0}, \\
& a_{p}=\frac{a_{P}^{\prime}}{\alpha}, c=c^{\prime}+\frac{a_{P}^{\prime}}{\alpha}(1-\alpha) \varphi_{P}^{0}, \\
& a_{P}=\sum b_{j P} \varphi_{j P}+c
\end{aligned}
$$




\section{Numerical calculation}

(1) The added mass coefficient of $\mathrm{R}=1$ sphere in unbounded flow field of the tetrahedron grid

Tetrahedron grid (Fig.3) and tetrahedron/prism hybrid grid (Fig.4) of R=1 sphere are generated by ICEM. For tetrahedron grid, there are 281261 tetrahedral elements and 6514 triangular elements on the sphere surface. For tetrahedron/prism hybrid grid, there are 253583 tetrahedral elements, 19008 triangular prism elements and 6336 triangles. The two grids were divided into eight parallel partitions by metis, and the relaxation iteration step is 3600 . The added mass calculated by tetrahedron grid are $\lambda_{11}=2.07994730, \lambda_{22}=2.08192865, \lambda_{33}=2.08158738$; added mass calculated by tetrahedron/prism hybrid grid are $\lambda_{11}=2.08840636, \lambda_{22}=2.09061189, \lambda_{33}=2.08996253$. The added mass coefficients of sphere by two grids are slightly different, and the accuracy of hybrid grid is slightly higher than that of tetrahedral. The calculation results of two grids agree with the theoretical results $2 \pi R^{3} / 3$ very well, and the maximum error is less than $0.65 \%$. It shows that the additional mass calculation method and program are viable.

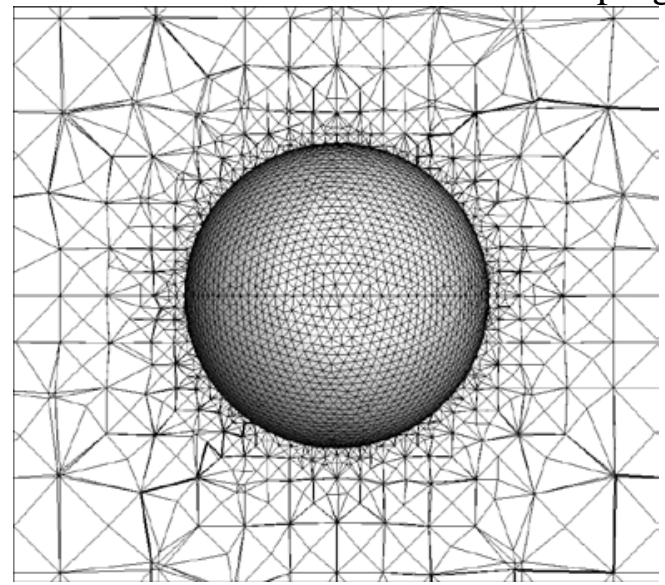

Fig.3. tetrahedron grid I

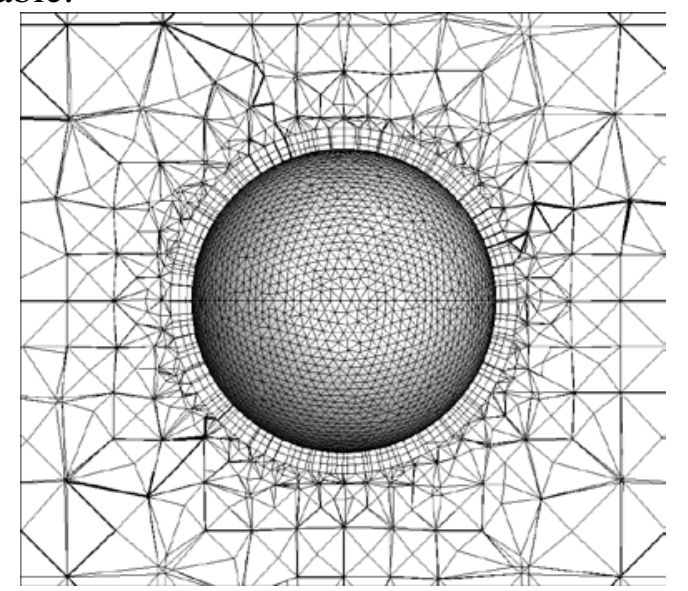

Fig.4. tetrahedron/prism hybrid grid II

(2) The added mass coefficients of a suspended platform in unbounded flow field

As shown in Fig.5, the shape structure of suspended platform is complex, which has not only a stable surface, but also a complex support structure, this means there will be relatively strong interference flow between the attachments. As shown in Fig.6, a platform grid of 5,179,432 tetrahedral elements is generated by ICEM. And then divided the grid into eight parallel partitions by metis, as shown in Fig.7 and Fig.8.

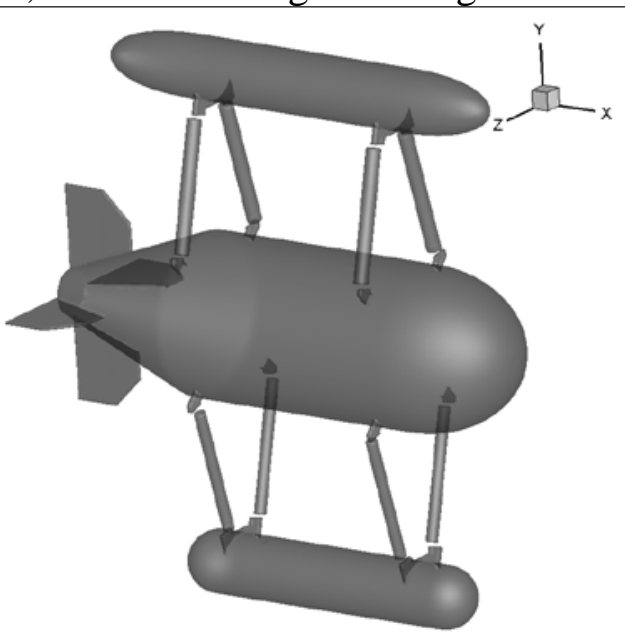

Fig.5. suspended platform shape

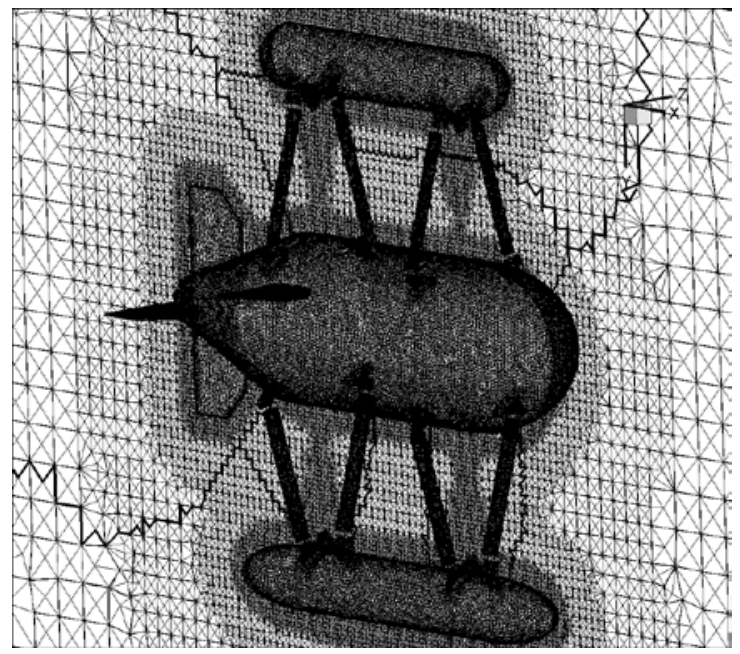

Fig.6. surface grid of suspended platform and partition boundaries of $\mathrm{z}=0$ cross section 


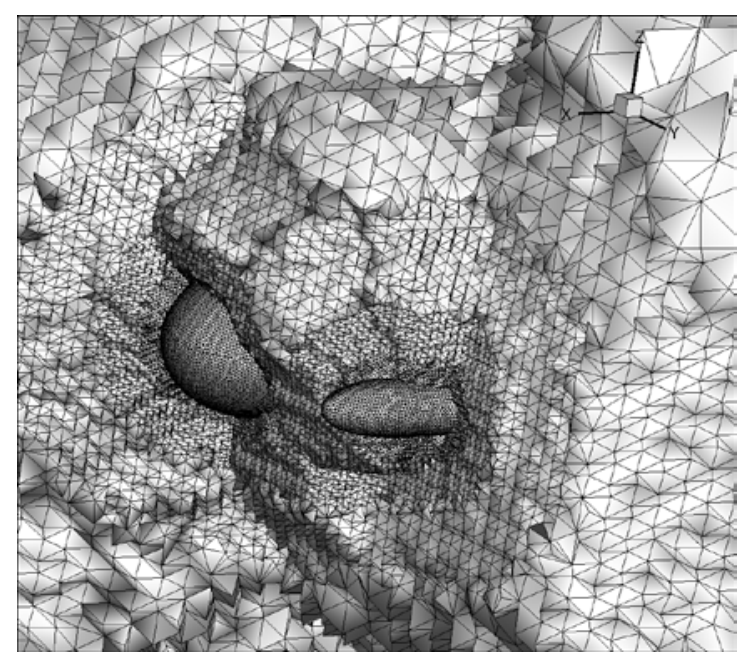

Fig. 7. parallel partition grid 1

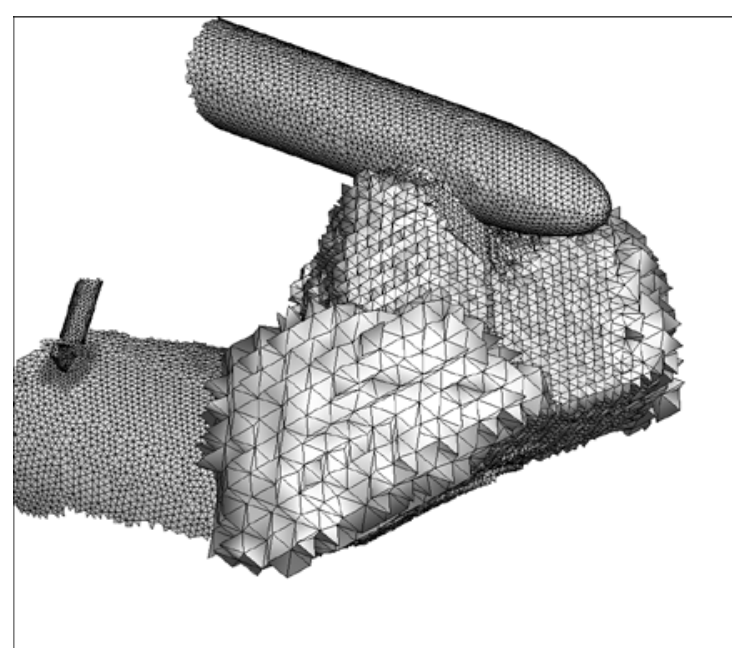

Fig. 8. parallel partition grid 2

Table 1 is the added mass coefficient of platform. In theory, the added mass coefficient of the platform is a symmetric 6 matrix, which means $\lambda_{i j}=\lambda_{j i}$.As shown in table $1,{ }^{{ }^{i j}}$ is slightly different from $\lambda_{j i}$, for plane symmetric platform, $\lambda_{i j}$ should be 0 in theory,such as $\lambda_{14}$ and $\lambda_{41}$. But these differences are due to incomplete symmetry grid of the three dimensional calculation domain and the numerical error in the solving process, and it is fully acceptable in engineering.

Table 1 The added mass coefficient $\lambda_{i j}$ of platform

\begin{tabular}{|c|c|c|c|c|c|c|}
\hline$\lambda$ & 1 & 2 & 3 & 4 & 5 & 6 \\
\hline 1 & 0.04428559 & 0.00003762 & 0.00000413 & -0.00000065 & -0.00000141 & 0.00007938 \\
\hline 2 & 0.00004035 & 0.25955725 & 0.00000758 & -0.00002628 & -0.00000790 & -0.01006647 \\
\hline 3 & 0.00001251 & -0.00000840 & 0.29400795 & 0.00831870 & 0.00785710 & 0.00000948 \\
\hline 4 & -0.00000092 & -0.00000123 & 0.00831783 & 0.05146063 & -0.00054964 & 0.00000110 \\
\hline 5 & -0.00000542 & -0.00000783 & 0.00784946 & -0.00054759 & 0.04824831 & -0.00000276 \\
\hline 6 & 0.00007919 & -0.01005780 & 0.00000770 & -0.00000061 & -0.00000683 & 0.05010740 \\
\hline
\end{tabular}

\section{Conclusion}

Based on the solution of Laplace equation in parallel using FVM with unstructured hybrid mesh , the paper puts forward an accurate and efficient calculation of added mass for underwater vehicle. Through calculating the added mass coefficients of a sphere and a suspended platform, it shows that the method has higher accuracy, and it is applicable to complex shape bodies with complex boundary conditions. Most importantly, this method is suitable for three-dimensional CFD program development, making position force, damping force and inertial force obtained by CFD calculation, so it has a strong practical significance.

\section{Acknowledgement}

In this paper, the research was sponsored by the Nature Science Foundation of discontinuous numerical algorithm( Project No.5012.111.10).

\section{References}

[1] John L. Hess and A.M.O. Smith. Calculation of non-lifting potential flow about arbitrary three-dimensional bodies [J], J. Ship Res., 1964, 8, 22-24

[2] John L. Hess and A.M.O. Smith. Calculation of potential flow about arbitrary bodies [J], Progress in Aeronautical Science, 1967,8, 1-138

[3] Y. J. Liu, S. Mukherjee, N. Nishimura, M.Schanz, W. Ye. A. Sutradhar, E. Pan, N .A. Dumont, 
A. Frangi, A. Saez. Recent Advances and Emerging Applications of the Boundary Element Method [J]. Applied Mechanics Reviews, Vol.64(2011), pp.1-38

[4] L. Landweber and M. Macagno, Irrotational flow about ship forms [R], IIHR Report No. 123, Iowa Institute of Hydraulic Research, The University of Iowa, Iowa City, Iowa, 1969.

[5] J. N. Newman and C.H. Lee, Boundary-element method in offshore structure analysis [J], Journal of Offshore Mech. And Arctic Eng. 124(May 2002), 81-89

[6] W. C. Webster, The flow about arbitrary, three-dimensional smooth bodies [J], Journal of Ship Research 19(4) (Dec. 1975), 206-218.

[7] Zhiliang Lin, Shijun Liao. Calculation of added mass coefficients of 3D complicated underwater bodies by FMBEM [J]. Communications in Nonlinear Science and Numerical Simulation. Volume 16, Issue 1, January 2011, Pages 187-194

[8] Li Baoyuan, Jiang Yuxi. Calculation of added mass for ship entering and leaving a ship-box [J]. Chinese Journal of Computational Mechanics. 2003,20(3),350-354.

[9] Wu xiuheng, Liu zuyuan, Shi shengda, Feng xueru. Ship Maneuverability [M]. Beijing, National Defense Industry Press, 2005, 285-286. 